\title{
Features of a single source describing the very end of the energy spectrum of cosmic rays
}

\author{
Alena Bakalová, ${ }^{a, *}$ Jakub Vícha ${ }^{a}$ and Petr Trávníček ${ }^{a}$ \\ ${ }^{a}$ FZU - Institute of Physics of the Czech Academy of Sciences, \\ Na Slovance 1999/2, Prague, Czech Republic \\ E-mail: bakalova@fzu.cz
}

The energy spectrum of cosmic rays extends over many orders of magnitude with a steep suppression of the flux at the highest energies. The energy spectrum of ultra-high energy cosmic rays (UHECR) is measured with great precision by the Pierre Auger Observatory and Telescope Array. However, the two measured spectra show different slopes of the suppression of the spectrum at the highest energies. This disagreement can be caused by the ability of these two experiments to see different parts of the sky and, therefore, in principle, different sources of UHECR as well. In our study, we investigate the possibility that the energy spectrum measured by the Pierre Auger Observatory at energies above $\log (E / \mathrm{eV}) \geq 19.5$ could be explained by a dominant single strong source. We explore the space of possible features of such a source including its distance, spectral index and mass composition, and compare the resulting flux after propagation using simulations in CRPropa 3 with the measured data. We show the possible parameters of such a source and explore possible mass composition mixes that could explain the data well.

$37^{\text {th }}$ International Cosmic Ray Conference (ICRC 2021)

July 12 th - 23rd, 2021

Online - Berlin, Germany

\footnotetext{
${ }^{*}$ Presenter
} 


\section{Introduction}

The very end of the energy spectrum of cosmic rays at ultra-high energies is measured with a high precision by two current experiments; the Pierre Auger Observatory [1] in the Southern hemisphere and Telescope Array [2] in the Northern hemisphere. Overall, the two measured spectra agree well, however, at the highest energies (above $\log (E / \mathrm{eV})=19.5)$ the spectrum measured by the Pierre Auger Observatory and Telescope Array exhibit different flux suppression as is shown on the left panel of Figure 1 [3]. Such disagreement might be caused by the ability of the two experiments to observe different sources on the sky in the Northern and Southern hemispheres that are able to accelerate cosmic rays up to the highest energies.

In this work, we investigate the possibility of a dominant strong source that would explain the structure of the end of the energy spectrum above $\log (E / \mathrm{eV})=19.5$ measured by the Pierre Auger Observatory. Furthermore, the measurements by the Pierre Auger Observatory $[4,5]$ might suggest that the mass composition at the highest energies is dominated by heavier elements with small to zero fraction of light elements in the composition mix as is depicted on the right panel of Figure 1. Even heavier mass composition at the highest energies can be interpreted using methods that assume ad-hoc adjustments to the predicted $X_{\max }$ distributions [6-8]. This additional condition allows us to further narrow the phase space of possible features of a possible dominant strong source.
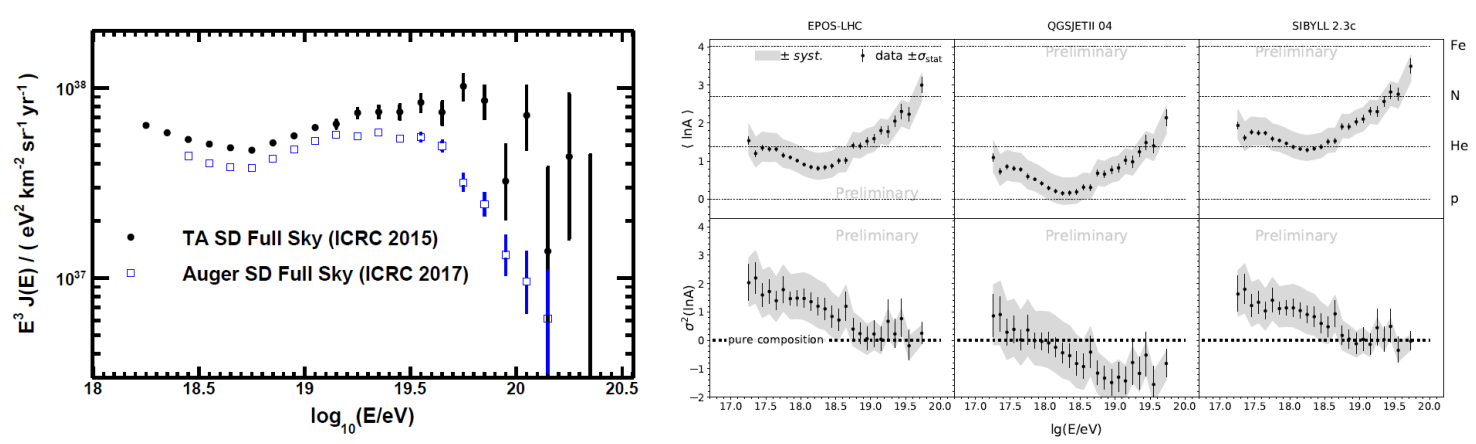

Figure 1: Left: measurements of the cosmic-ray energy spectrum by the Pierre Auger Observatory and Telescope Array [3]. Right: moments of $\ln A$ distributions from measurements of depth of shower maximum at the Pierre Auger Observatory interpreted using different hadronic interaction models [4].

\section{Simulations and Data}

To investigate a possible single-source scenario explaining the very end of the cosmic-ray energy spectrum, we simulate propagation of cosmic rays in a one-dimensional mode of CRPropa 3 [9]. A single source is considered at distances from $3 \mathrm{Mpc}$ up to $100 \mathrm{Mpc}$ with a step of $1 \mathrm{Mpc}$ up to $10 \mathrm{Mpc}$ and a step of $10 \mathrm{Mpc}$ farther of. We assume four types of injected nuclei at the source: proton, helium $\left({ }^{4} \mathrm{He}\right)$, nitrogen $\left({ }^{14} \mathrm{~N}\right)$ and iron $\left({ }^{56} \mathrm{Fe}\right)$. We inject 10,000 particles for each primary and source distance with a power-law energy spectrum with spectral index $\gamma=2$ with energy from $\log _{10}(E / \mathrm{eV})=19.5$ up to a maximal rigidity of $\log _{10}\left(R_{\max } / \mathrm{V}\right)=20.5$. During the simulation of propagation, individual particles undergo energy-loss processes (electron-pair production, photopion production, photodisintegration) due to interactions with cosmic microwave background and 
extragalactic background light together with redshift effects and nuclear decays in case of unstable isotopes.

To investigate potential sources with capability to accelerate particles to different maximum energies, we apply a broken-rigidity exponential cutoff to the energy spectrum as

$$
\frac{\mathrm{d} N}{\mathrm{~d} E}=E^{-\gamma} \cdot f_{\text {cut }},
$$

where $\gamma$ is the spectral index and $f_{\text {cut }}$ is the rigidity exponential cutoff function defined for a given rigidity cutoff $R_{\text {cut }}$ as

$$
f_{\text {cut }}=\left\{\begin{array}{ll}
1 & \left(E<Z e R_{\text {cut }}\right) \\
e^{\left(1-\frac{E}{Z e R_{\text {cut }}}\right)} & \left(E>Z e R_{\text {cut }}\right)
\end{array},\right.
$$

where $\mathrm{Z}$ is the proton number of the primary particle, see e.g. [11]. We investigate the rigidity cutoff from $\log _{10}\left(R_{\text {cut }} / \mathrm{V}\right)=20.5$ with discrete steps of 0.1 down to $\log _{10}\left(R_{\text {cut }} / \mathrm{V}\right)=18.5$ where the energy spectrum at the highest energies is then dominated by the exponential behavior of the generated energy spectrum of all primaries. The energy spectrum is also reweighted to follow different spectral indices in the range $\gamma \in(1,3)$ with a step of 0.5 . We mix the primary particles with a step of $10 \%$ in the relative fraction making total of 286 possible mass composition scenarios on the source.

The energy spectrum obtained from the simulations for different mass composition scenarios, spectral index, rigidity cutoff and source distance is compared with the shape of the energy spectrum measured by the Pierre Auger Observatory in the range of logarithm of energy $19.5-20.2$. The data measured by the Pierre Auger Observatory that were used in this work are listed in Table 1.

We define a good agreement between the measured and simulated energy spectra with a condition of $\chi^{2} / \mathrm{ndf}<2$. Another condition that must be fulfilled is that the simulated spectrum must contain particles in all investigated energy bins.

\begin{tabular}{|c|c|}
\hline $\log _{10}(E / \mathrm{eV})$ & $J\left[\mathrm{~km}^{-2} \mathrm{yr}^{-1} \mathrm{sr}^{-1} \mathrm{eV}^{-1}\right]$ \\
\hline \hline 19.55 & $\left(1.252_{-0.050}^{+0.052}\right) \cdot 10^{-21}$ \\
\hline 19.65 & $\left(5.98_{-0.31}^{+0.32}\right) \cdot 10^{-22}$ \\
\hline 19.75 & $\left(1.93_{-0.15}^{+0.17}\right) \cdot 10^{-22}$ \\
\hline 19.85 & $\left(8.10_{-0.88}^{+0.99}\right) \cdot 10^{-23}$ \\
\hline 19.95 & $\left(1.86_{-0.38}^{+0.46}\right) \cdot 10^{-23}$ \\
\hline 20.05 & $\left(5.5_{-1.8}^{+2.5}\right) \cdot 10^{-24}$ \\
\hline 20.15 & $\left(2.9_{-1.2}^{+1.7}\right) \cdot 10^{-24}$ \\
\hline
\end{tabular}

Table 1: Data of cosmic-ray flux in energy bins $\log _{10}(E / \mathrm{eV})$ with a step of 0.1 at the highest energies measured by the Pierre Auger Observatory [10]. 


\section{Results}

Comparing the simulated energy spectra produced by sources with distances from $3 \mathrm{Mpc}$ up to $100 \mathrm{Mpc}$, different spectral indices, mass composition mixes and maximum rigidity cutoffs with the measured energy spectrum shown in Table 1 we find possible source features describing the spectrum well for all investigated rigidity cutoffs $R_{\text {cut }}$ and spectral indices. These two features of the source are closely connected as only some combinations of these variables can produce a spectrum compatible with the measurement.

A minimal value of $\chi^{2} /$ ndf for different $R_{\text {cut }}$ and spectral indices $\gamma$ of the source are shown in Figure 2. The regions with darker contours represent combinations of the rigidity cutoff and spectral index of the source that can produce an energy spectrum compatible with the measured one. Sources with low spectral indices $(\gamma=1, \gamma=1.5)$ are able to describe the measured energy spectrum well only in a narrow band of $R_{\text {cut }}$ values, while sources with higher spectral indices can describe the energy spectrum on the Earth within the whole range of different $R_{\text {cut }}$ values as the spectral index on the source is closer to the one on the Earth. Concerning the parameter of the source distance, all possible solutions that were found with a good agreement are for sources within $50 \mathrm{Mpc}$ from the Earth.

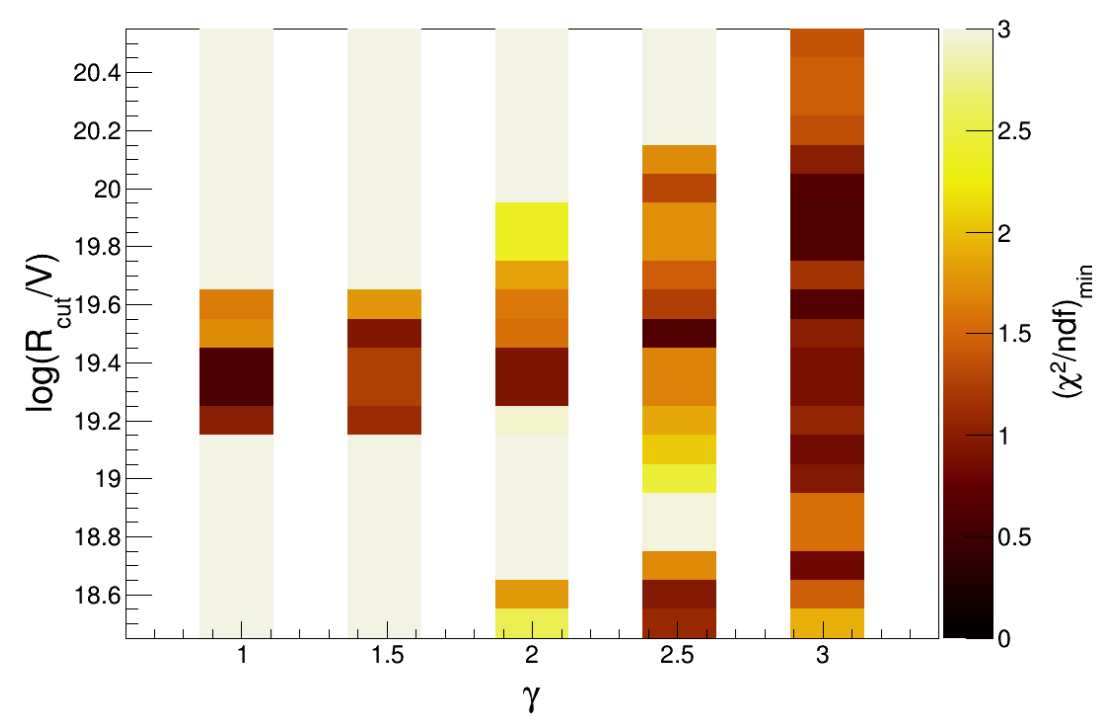

Figure 2: A minimal value of $\chi^{2} /$ ndf for sources with different rigidity cutoffs $R_{\text {cut }}$ and spectral indices $\gamma$. Minimal $\chi^{2} /$ ndf is scanned over all source distances and mass composition mixes on the source.

For all possible solutions, the mean mass number on the Earth $\langle\ln A\rangle_{\text {Earth }}$ for different $R_{\text {cut }}$ is shown in Figure 3 together with the variance $\sigma^{2}\left(\ln A_{\text {Earth }}\right)$. A source with $\log _{10}\left(R_{\text {cut }} / \mathrm{V}\right) \leq 19.2$ is producing rather heavy mass composition mixes on the Earth, while a source with higher rigidity cutoff can produce mass composition mixes containing light elements as well.

To further narrow the possible source features, besides of the shape of the energy spectrum above $\log _{10}(E / \mathrm{eV})=19.5$, we include an additional condition on the mass composition of the cosmic rays on the Earth based on available measurements. On the right panel of Figure 1, the interpretation of the shower maximum measurement by the Pierre Auger Observatory is shown for 

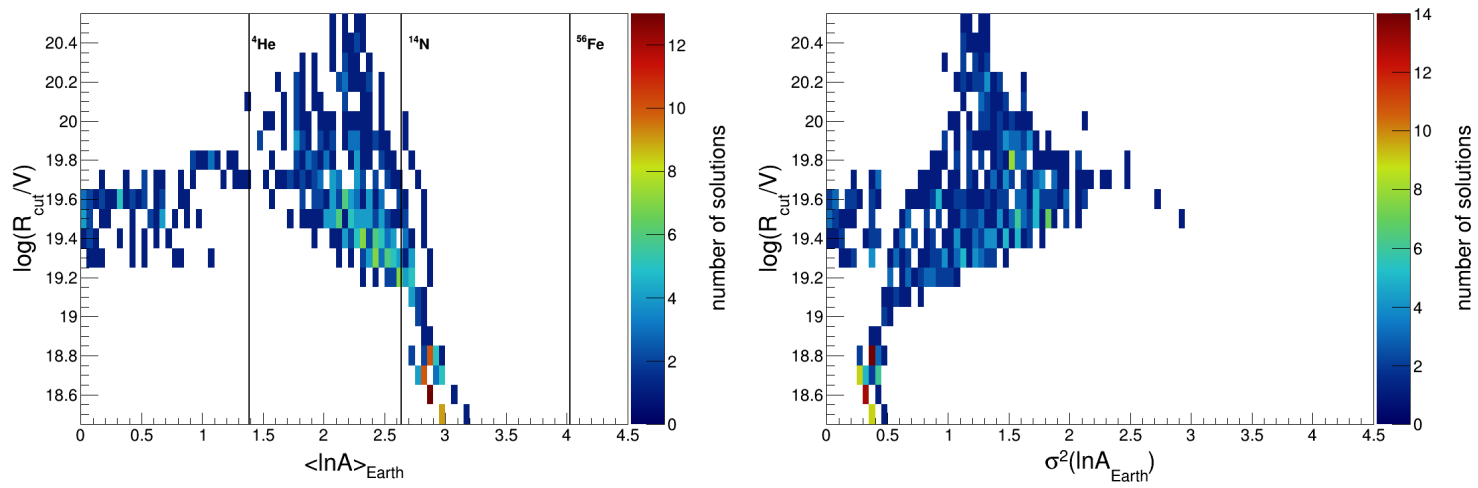

Figure 3: Left: mean mass number on the Earth for sources with different rigidity cutoff $R_{\text {cut }}$ producing an energy spectrum compatible with the measured spectrum. Right: variance of the mass number on the Earth for individual sources with different rigidity cutoff $R_{\text {cut }}$ producing an energy spectrum compatible with the measured spectrum.
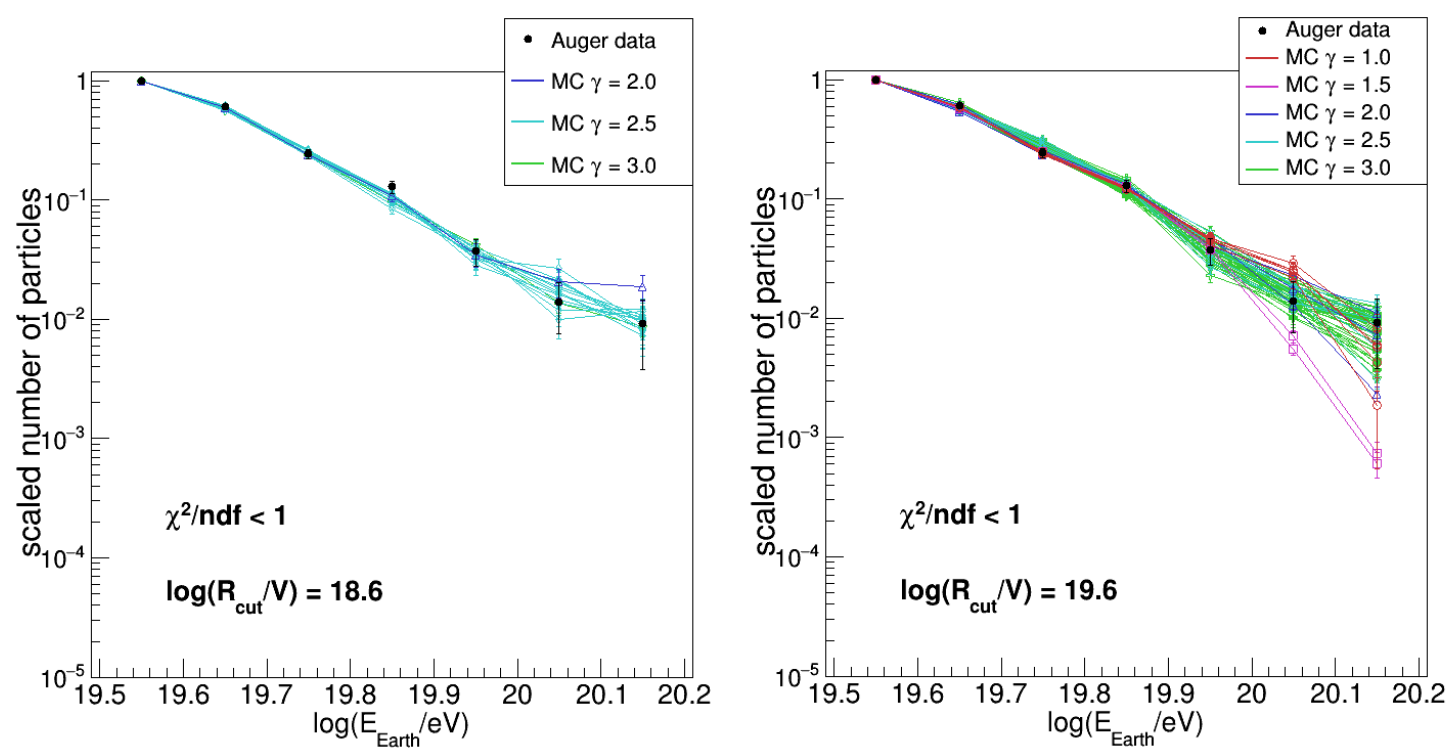

Figure 4: Simulated energy spectra compatible with the measured one with $\chi^{2} /$ ndf $<2$ for two rigidity cutoffs on the source; $\log _{10}\left(R_{\text {cut }} / \mathrm{V}\right)=18.6($ left $)$ and $\log _{10}\left(R_{\text {cut }} / \mathrm{V}\right)=19.6$ (right).

different hadronic interaction models. In the case of EPOS-LHC and Sybill 2.3c, the measured data may suggest a heavy mass composition at the highest energies, which was supported by the Delta method using measurements at the ground level [5]. Therefore, we choose to look for source features with $\langle\ln A\rangle_{\text {Earth }}$ larger than for nitrogen nuclei together with $\sigma^{2}(\ln A)_{\text {Earth }} \leq 0.5$. We do not consider the interpretation of the data using QGSJet II-04 from Figure 1 as the model predicts negative variances of $\ln A_{\text {Earth }}$.

To satisfy these conditions on the mass composition on the Earth, we found that only solutions for sources with $\log _{10}\left(R_{\text {cut }} / \mathrm{V}\right)<19.2$ can produce compatible both energy spectrum and composition mixes. Furthermore, only sources with spectral index of the energy spectrum $\gamma \geq 2$ are satisfying both conditions. The energy spectra for $\log _{10}\left(R_{\text {cut }} / \mathrm{V}\right)=18.6$ and $\log _{10}\left(R_{\text {cut }} / \mathrm{V}\right)=19.6$ 
are shown in Figure 4 on the left and right panel, respectively. The predicted mass compositions on the Earth are depicted in Figures 5 and 6 for the two different rigidity cutoffs, respectively. In Figure 4 we can see that the energy spectrum is well described for both rigidity cutoffs and some spectral indices of the source. However, Figures 5 and 6 show different energy evolutions of the $\langle\ln A\rangle_{\text {Earth }}$ and $\sigma^{2}(\ln A)_{\text {Earth }}$ for these two rigidities. For a source with lower rigidity, we not only describe the shape of the spectrum well but also the mass composition requirements are satisfied. For a source with the higher rigidity cutoff, the mass composition on the Earth is lighter and has a large variance, meaning a large fraction of lighter elements in it. The reason for the heavy composition at low $R_{\text {cut }}$ is the suppression of light elements on the source itself, therefore predominantly heavy elements are created in the investigated energy range and subsequently "detected" on the Earth. To satisfy the mass composition conditions, the source should be within $10 \mathrm{Mpc}$ from the Earth.
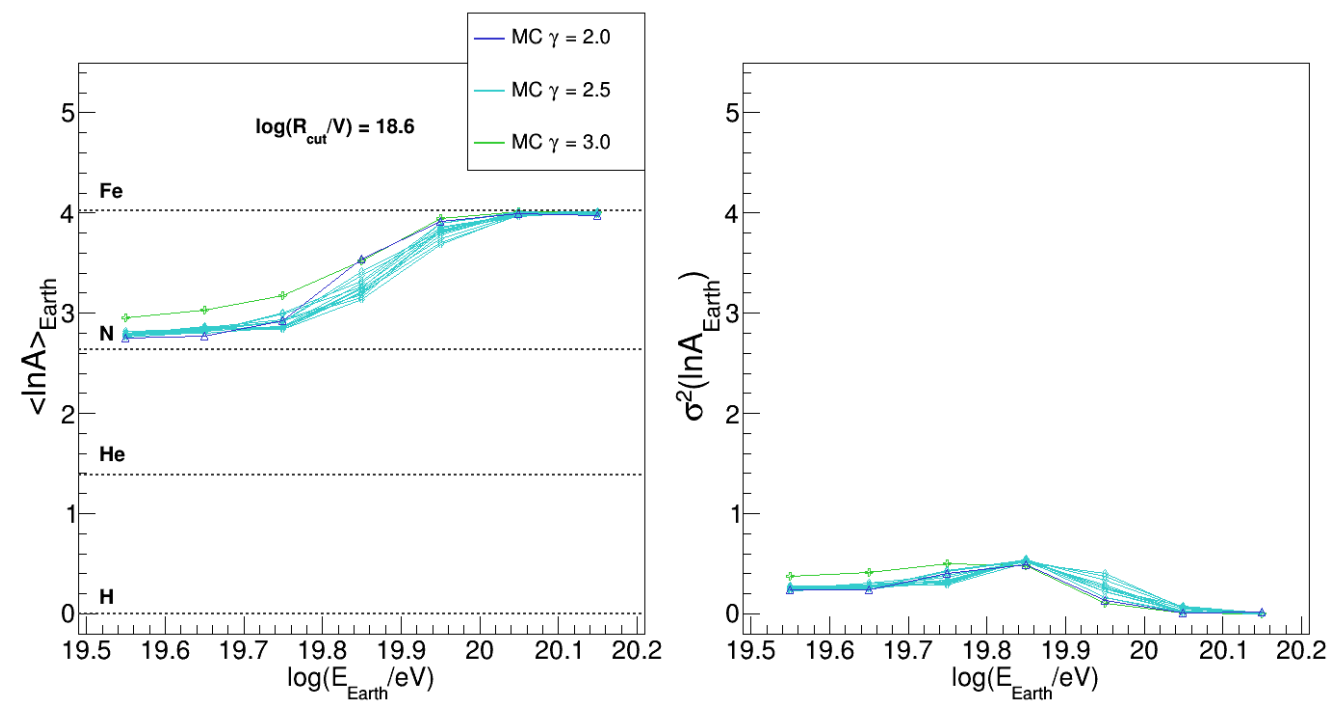

Figure 5: Energy evolution of the mean (left) and variance (right) of $\ln A$ on the Earth for solutions compatible with the shape of the energy spectrum for a source with $\log \left(R_{\text {cut }} / \mathrm{V}\right)=18.6$.

Finally, we want to stress out that in case of a dominant source contributing to the energy spectrum at the highest energies with such a heavy mass composition, the arrival directions of cosmic rays on the Earth would not create a point-like anisotropies. The Galactic magnetic field (GMF) has a significant influence on particles with low rigidities (for Fe primary with energy $\log _{10}(E / \mathrm{eV})=19.5$ the corresponding rigidity is $\left.\log _{10}(R / \mathrm{V}) \sim 18.1\right)$. An example of arrival directions of cosmic rays with rigidity $\log _{10}(R / \mathrm{V})=18.1$ on Earth after simulated propagation in a JF12 model of the GMF [12] is depicted in Figure 7 for three different locations of an extragalactic source. The GMF isotropises the arrival directions of cosmic rays and therefore at such low rigidities the information about the source would be lost during the propagation in the GMF.

\section{Summary}

We investigated parameters of a single dominant source that can produce an energy spectrum of cosmic rays above $\log _{10}(E / \mathrm{eV})=19.5$ compatible with the energy spectrum measured by the Pierre 

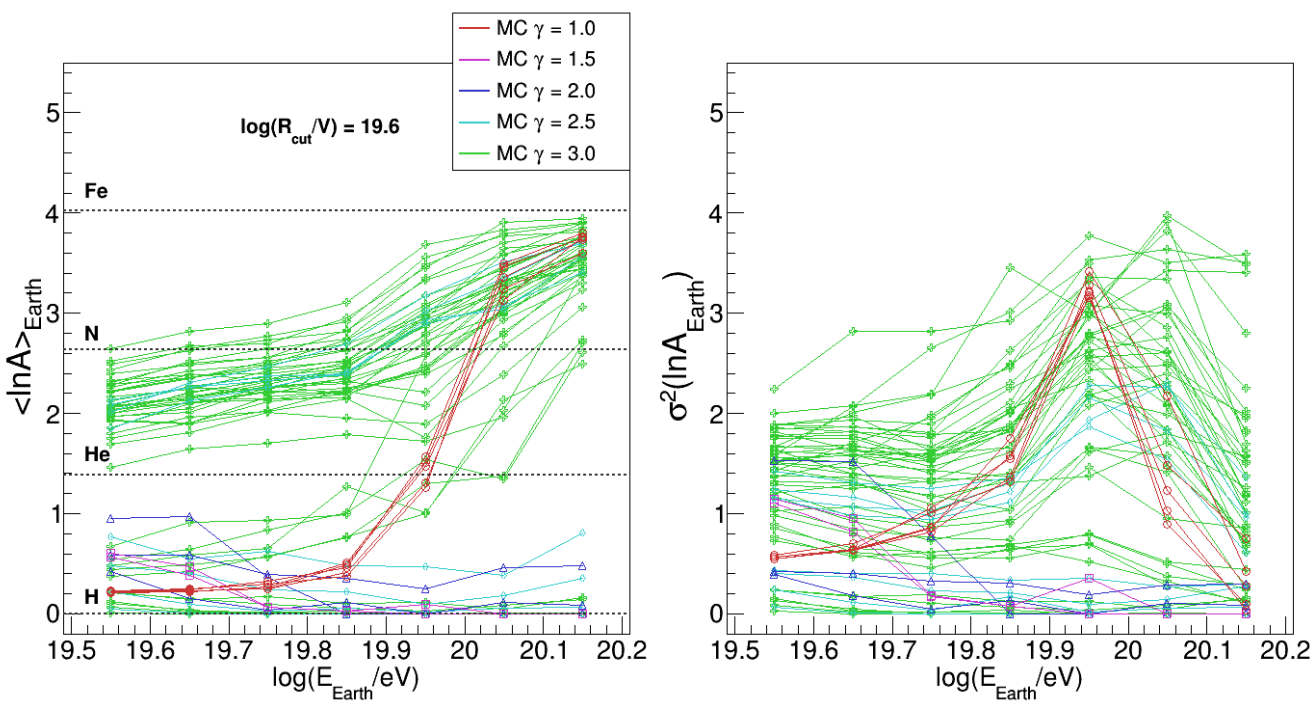

Figure 6: Energy evolution of the mean (left) and variance (right) of $\ln A$ on the Earth for solutions compatible with the shape of the energy spectrum for a source with $\log \left(R_{\text {cut }} / \mathrm{V}\right)=19.6$.

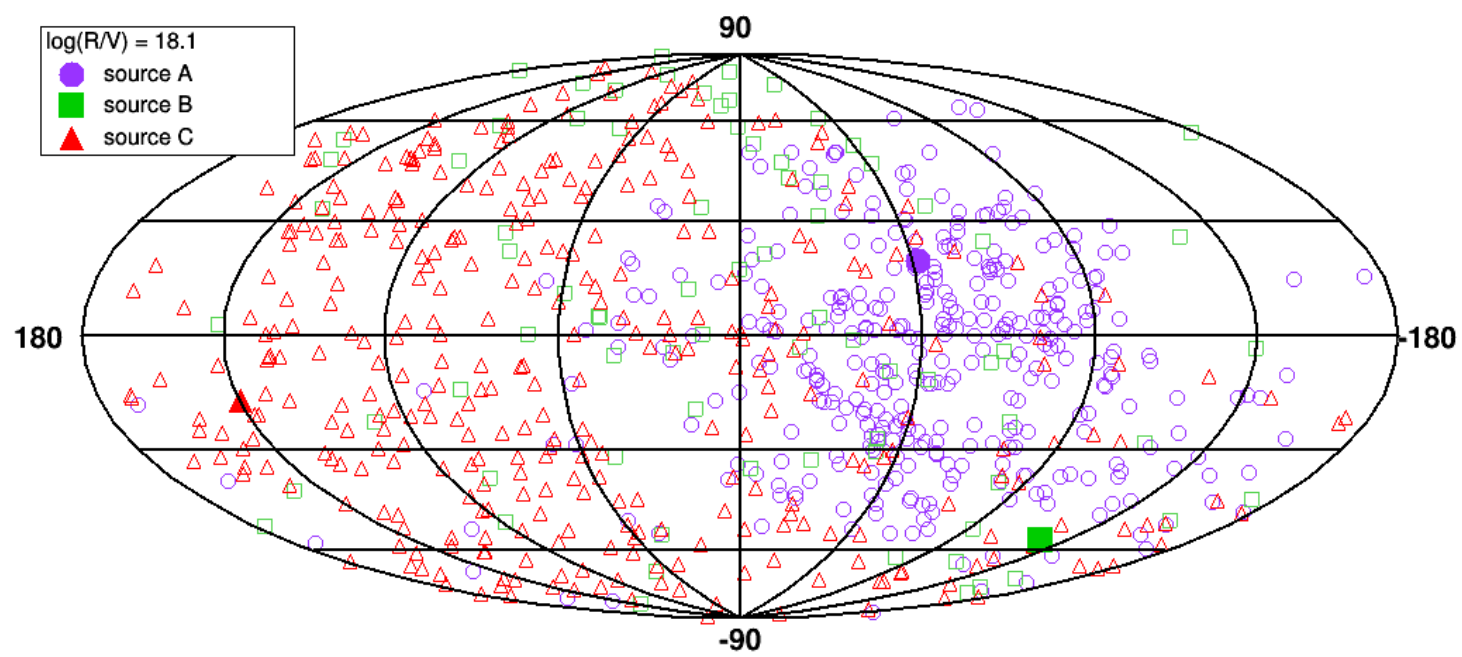

Figure 7: Arrival direction of cosmic rays with $\log (R / \mathrm{V})=18.1$ on the Earth propagated in the JF12 model of GMF originating from three different example directions of the extragalactic sources. The skymap is in Galactic coordinates.

Auger Observatory with $\chi^{2} /$ ndf $<2$. Using one-dimensional simulations in CRPropa 3 simulation framework, we show that a source within $50 \mathrm{Mpc}$ from Earth can produce such an energy spectrum with various parameters of the spectral index, particles composition and maximum rigidity cutoff. When including another conditions on the mass composition on the Earth, based on results from the Pierre Auger Observatory published in [4], [5] and [6], we can further narrow the space of the possible source features.

Using the assumed conditions on heavy composition on the Earth $(<\ln A>$ above the one 
for nitrogen) and small variance $\left(\sigma^{2}(\ln A)_{\text {Earth }} \leq 0.5\right)$, we find that a single dominant source can produce an energy spectrum compatible with the observed one only for lower rigidity cutoffs $\log _{10}(R / \mathrm{V})<19.2$ and spectral indices $\gamma \geq 2$ of the source. Moreover, the distance of the source should be within $10 \mathrm{Mpc}$ from the Earth. Finally, we want to note that a single dominant source with heavy mass composition would not necessarily cause a point-like anisotropy in arrival directions of UHECRs due to the isotropisation of the arrival directions in the GMF.

\section{Acknowledgments}

This work is funded by the Czech Republic grants of MEYS CR: LTT18004, LM2015038, LM2018102, CZ.02.1.01/0.0/0.0/16_013/0001402, CZ.02.1.01/0.0/0.0/18_046/0016010.

\section{References}

[1] A. Aab et al. [Pierre Auger Collaboration], Nucl. Instrum. Meth. A 798 (2015) 172.

[2] H. Kawai, S. Yoshida, H. Yoshii et al., Nuclear Physics B Proceedings Supplements 175 (2008) 221-226.

[3] T. AbuZayyad, O. Deligny, D. Ikeda, D. et al., European Physical Journal Web of Conferences 210 (2019) 01002.

[4] A. Yushkov for the Pierre Auger Collaboration, Proceedings of 36th International Cosmic Ray Conference - PoS(ICRC2019) 358 (2019) 482.

[5] C. J. T. Peixoto for the Pierre Auger Collaboration, Proceedings of 36th International Cosmic Ray Conference — PoS(ICRC2019), 358 (2019) 440.

[6] S. Blaess, J.A. Bellido, B.R. Dawson, arXiv (2018) arXiv:1803.02520.

[7] J. Vicha for the Pierre Auger Collaboration, Proceedings of 37th International Cosmic Ray Conference - PoS(ICRC2021), 395 (2021) 310.

[8] J. Glombitza for the Pierre Auger Collaboration, Proceedings of 37th International Cosmic Ray Conference -PoS(ICRC2021), 395 (2021) 359.

[9] R. Alves Batista et al., JCAP 05 (2016) 038.

[10] A. Aab et al. [Pierre Auger Collaboration], Phys. Rev. D 102 (2020) 062005.

[11] The Pierre Auger Collaboration, JCAP 04 (2017) 038.

[12] R. Jansson and G. R. Farrar, ApJ 757 (2012) 14. 